\title{
Electronic property analysis of O-doped $\mathrm{Cu}_{3} \mathrm{SbS}_{3}$
}

\author{
C. Tablero
}

\begin{abstract}
A B S T R A C T
The ternary $\mathrm{Cu}-\mathrm{Sb}-\mathrm{S}$ semiconductors are considered to be sustainable and potential alternative absorber materials in thin film photovoltaic applications. In these compounds, several phases may coexist, albeit in different proportions depending on experimental growth conditions. Additionally, the photovoltaic efficiency could be increased with isoelectronic doping. In this work we analyze the electronic properties of O-doped $\mathrm{Cu}_{3} \mathrm{SbS}_{3}$ in two structures: the wittichenite and the skinnerite. We use first-principles within the density functional formalism with two different exchange-correlation potentials. In addition, we estimate the potential of these compounds for photovoltaic applications.
\end{abstract}

\section{Introduction}

The ternary system $\mathrm{Cu}_{x} \mathrm{Sb}_{y} \mathrm{~S}_{z}$ is considered to be a potential low-cost sustainable absorber material for thin film solar cells due to the availability and low cost of the constituent elements. The ternary phases in this system are: $\mathrm{Cu}_{3} \mathrm{SbS}_{4}$ (famatinite), $\mathrm{CuSbS}_{2}$ (chalcostibite), $\mathrm{Cu}_{3} \mathrm{SbS}_{3}$ (skinnerite and wittichenite) and $\mathrm{Cu}_{12} \mathrm{Sb}_{4} \mathrm{~S}_{13}$ (tetrahedrite). Thin films of $\mathrm{CuSbS}_{2}$ have been deposited using a range of different techniques, such as spray pyrolysis [1], direct evaporation [2] and chemical bath deposition followed by annealing $[3,4]$. Using the last technique, $\mathrm{Cu}_{3} \mathrm{SbS}_{3}$ has also been deposited [4]. Particles of ternary $\mathrm{Cu}_{3} \mathrm{SbS}_{4}$ films were prepared using the Polyol-Route [5], and high quality semiconducting famatinite nanofibers and tetradrite nanoflakes have been selectively synthesized using the mild hydrothermal and solvothermal synthesis route [6].

It is experimentally difficult to control the phase and impurity concentration of these materials. In general, there are several phases in the experimental samples, albeit in different proportions. Some of the ternary phases have been partially explored. $\mathrm{The} \mathrm{Cu}_{3} \mathrm{SbS}_{4}$ gap presented in the literature is between $0.46 \mathrm{eV}$ [7] and $0.74 \mathrm{eV}$ [8]. $\mathrm{CuSbS}_{2}$ appears to be a direct band-gap semiconductor [9] with a band-gap energy between $1.38 \mathrm{eV} \mathrm{[10]} \mathrm{and}$ $1.5 \mathrm{eV}$ [3]. $\mathrm{Cu}_{3} \mathrm{SbS}_{3}$ and $\mathrm{Cu}_{12} \mathrm{Sb}_{4} \mathrm{~S}_{13}$ exhibit optical bandgaps of $1.60 \mathrm{eV}$ [3] and $1.2 \mathrm{eV}$ [11], respectively. The gaps of the last three phases are close to the optimum value required for solar energy conversion $(-1.4 \mathrm{eV})$.

Additionally, doping II-VI compound semiconductors with oxygen has been shown to give rise to deep traps [12-15] at which carriers recombine radiatively $[16,17]$. The insertion of intermediate states within the bandgap of a semiconductor material provides additional paths for optical transitions creating the possibility for extremely high efficiency ( $>60 \%$ ) [18].

$\mathrm{Cu}_{3} \mathrm{SbS}_{3}$ is a promising material for solar cell applications. In order to analyze its potentiality as a photovoltaic absorbent material for thin film structures, we present a study of the electronic properties of O-doped $\mathrm{Cu}_{3} \mathrm{SbS}_{3}$ using first-principles with two different exchange-correlation potentials. We focus our analysis on two polymorphic structures. Knowing the differences between these structures will be very useful for optoelectronic applications.

\section{Calculations}

\subsection{Total and single-particle energies}

A first-principles methodology within the density functional formalism $[19,20]$ is used to analyze how the incorporation of isoelectronic $\mathrm{O}$ impurities on substitutional $\mathrm{S}$ sites of $\mathrm{Cu}_{3} \mathrm{SbS}_{3}$ influences its electronic and optical properties. For the exchangecorrelation potential, we have employed the generalized gradient approximation (GGA) in the form of Perdew, Burke and Ernzerhof [21], and the Local Density approximation (LDA) with the Perdew-Zunger parametrization to the Ceperley-Alder numerical data [22]. The standard Troullier-Martins [23] pseudopotentials are adopted and expressed in the Kleinman-Bylander [24] factorized form. The valence wave functions are expanded in a numerically localized pseudoatomic orbital basis set [25].

We have used two different strategies to obtain the band edges and transition-energy levels at experimentally determined lattice constants: single-particle band structure calculations and total 
a

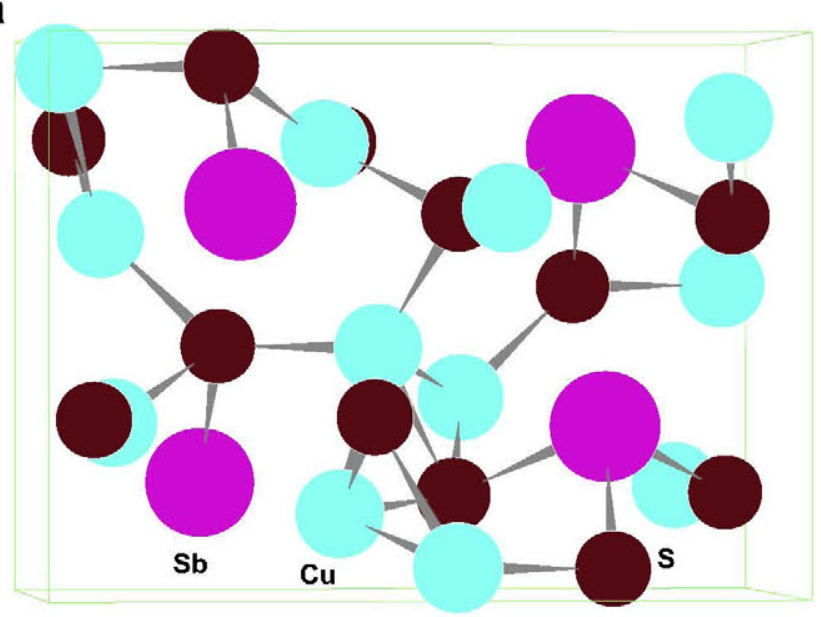

b



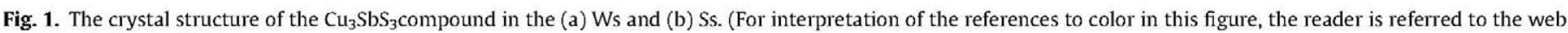
version of this article.)

a

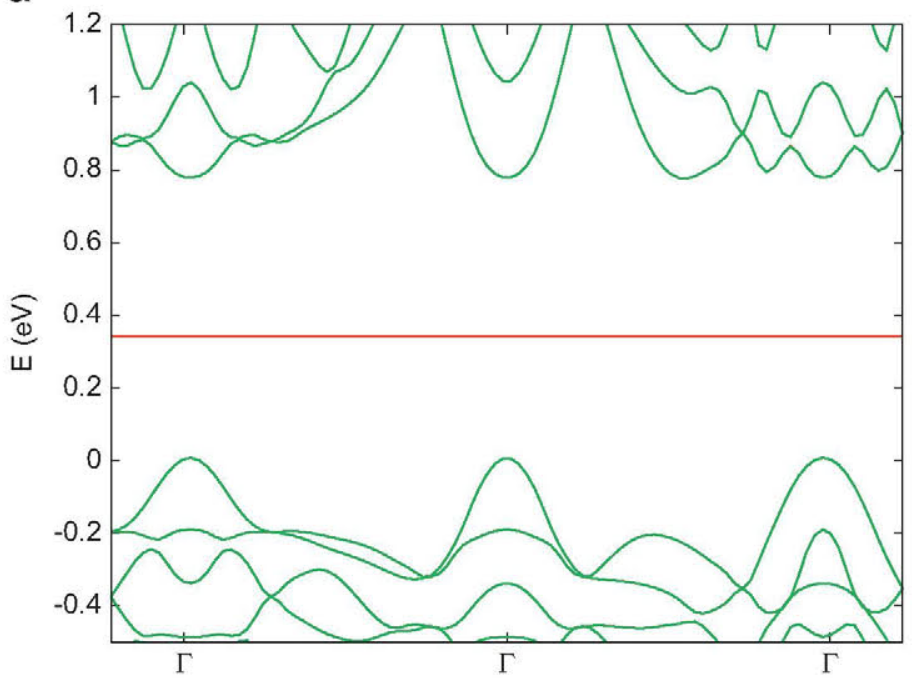

b



C

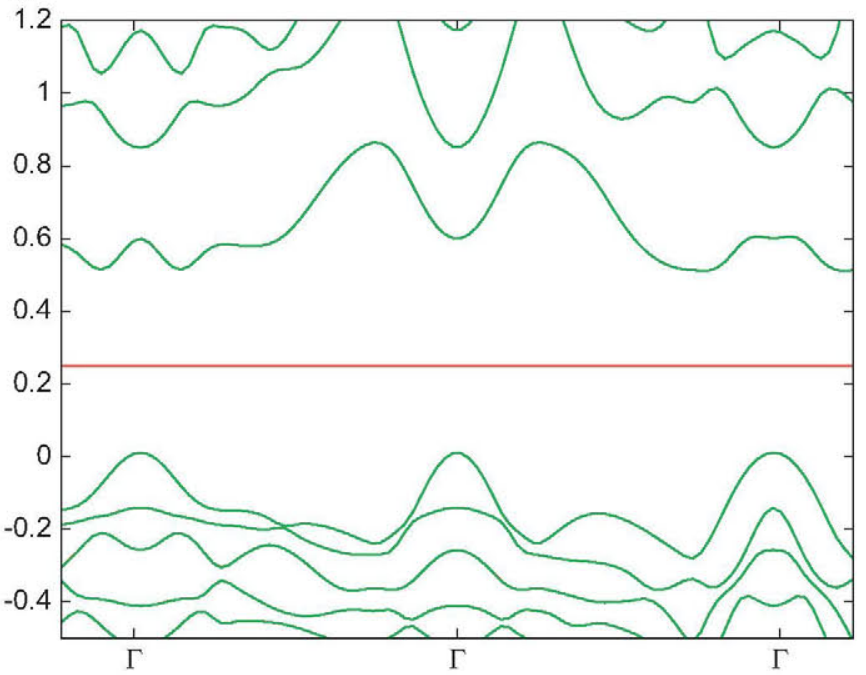

d

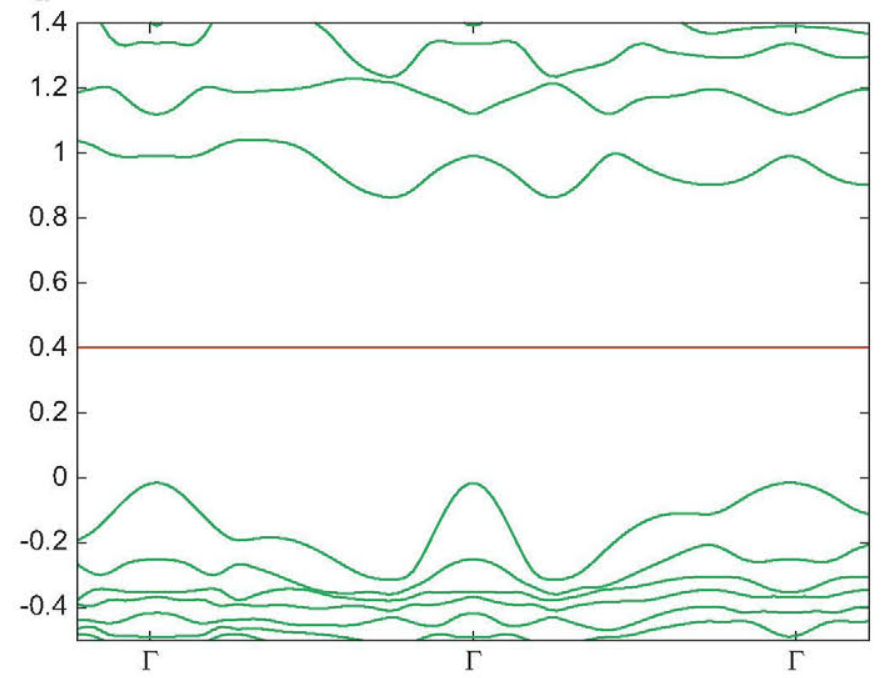

Fig. 2. Energy-band diagram with GGA for $\mathrm{Cu}_{3} \mathrm{SbS}_{3}$ in the (a) Ws and (b) Ss, and for O-doped $\mathrm{Cu}_{3} \mathrm{SbS}_{3}$ in the (c) Ws and (d) Ss. The VB edge has been chosen as the energy origin. 
defect formation energies calculations. Using LDA and GGA and taking the gap to be the difference between single-particle band structure energies is known to lead to its underestimation due to correlation problems. Using differences of single-particle band structure energies is equivalent to using Koopman's theorem. Nevertheless, if total defect formation energies [26,27] are used instead of the single-particle energies, correlation problems and systematic errors are decreased [28].

To determine the optical properties, the momentum matrix elements of the single-particle band structure have been calculated. From these, and the energy and occupations of the bands at $\vec{k}$ points of the Brillouin zone, the absorption coefficients are obtained.

\subsection{Crystal structure}

$\mathrm{Cu}_{3} \mathrm{SbS}_{3}$ is polymorphic. Below $263 \mathrm{~K}, \mathrm{Cu}_{3} \mathrm{SbS}_{3}$ has the Wittichenite structure (Ws) [29] (Fig. 1a), the low temperature form of $\mathrm{Cu}_{3} \mathrm{BiS}_{3}$. The space group of the Ws is $P 2_{1} 2_{1} 2_{1}$ (No. 19) with lattice parameters $a=7.884(1) \AA, \quad b=10.221(1) \AA$, $c=6.624(1) \AA$ [30]. Between 263 and $395 \mathrm{~K}, \mathrm{Cu}_{3} \mathrm{SbS}_{3}$ has the Skinnerite structure (Ss) [30-32] (Fig. 1b). This is monoclinic, with the space group $P 2_{1} / \mathrm{c}$ (No. 14). Above $395 \mathrm{~K}$, the $\mathrm{Cu}_{3} \mathrm{SbS}_{3}$ crystallizes in the orthorhombic system [30,31], with space group Pnma (No. 62) and lattice parameters $a=7.808(1) \AA, b=10.252$ (2) $\AA, c=6.587(2) \AA$. This $\alpha-\mathrm{Cu}_{3} \mathrm{SbS}_{3}$ high-temperature modification is similar to the high temperature $\mathrm{Cu}_{3} \mathrm{BiS}_{3}$ structure.

In the Ws, the $\mathrm{Cu}$ is in nearly trigonal planar coordination with $\mathrm{S}, \mathrm{Sb}$ is trigonally coordinated by $\mathrm{S}$, and $\mathrm{S}$ is tetrahedrally coordinated by three copper atoms and one antimony atom. In the $\mathrm{Ss}$, the $\mathrm{Sb}$ atom has trigonal pyramidal coordination with $\mathrm{S}$, and most $\mathrm{Cu}$ sites have triangular coordination with $\mathrm{S}$ atoms. There are two different positions for the $\mathrm{Cu}(\mathrm{Cu}(\mathrm{a})$ and $\mathrm{Cu}(\mathrm{b}))$ (red and yellow in Fig. 1(b) which cannot be occupied simultaneously, since this would result in the distance between these positions being so small as to result in an energetically unstable structure. This implies the need to average the results over numerous supercells. We have analyzed a sample of supercells and the energy differences are inside the margin of the error of the calculations.

\section{Results and disscusion}

Applying the aforementioned calculation method to the aforementioned crystal structures, we have obtained the corresponding single-particle band energies and total energies. For the host $\mathrm{Cu}_{3} \mathrm{SbS}_{3}$ in the Ws, the gaps obtained by taking the difference between the single-particle band energies are $0.66 \mathrm{eV}$ and $0.77 \mathrm{eV}$ for LDA and GGA respectively. For the Ss, the gaps are $0.87 \mathrm{eV}$ (LDA) and $1.00 \mathrm{eV}$ (GGA). Using total defect formation energies instead of the single-particle energies to reduce correlation problems and systematic errors, the gaps obtained are: $1.03 \mathrm{eV}$ (Ws-LDA), $1.12 \mathrm{eV}$ (Ws-GGA), $1.19 \mathrm{eV}$ (Ss-LDA), and $1.31 \mathrm{eV}$ (Ss-GGA). These values, although still affected by the LDA/GGA underestimation, compare well with experimental results in the literature (1.5-1.6 eV [4]).

In Fig. 2, the GGA calculated band structures of host and the $\mathrm{O}$-doped $\mathrm{Cu}_{3} \mathrm{SbS}_{3}$ in the Ws and in the Ws are shown. The $\mathrm{O}$ doping separates a sub-band from the conduction band, which is pushed deeper into the gap. The Fermi energy (horizontal line in the figure) is between the valence band (VB) and the sub-band, indicating that it has an acceptor character.

In order to identify the atomic contribution of the $\mathrm{O}$ atom to the deeper sub-band, the atomic projected density of states (DOS) per atom is represented in Fig. 3. The 0 is tetrahedrally coordinated by three copper atoms and one antimony atom. Therefore, the oxygen atoms will interact mainly with their nearest neighbors. The deeper sub-band at the $\mathrm{CB}$ edge is essentially constituted by the combination of $\mathrm{O}$ and the $\mathrm{Sb}$ orbitals. An additional analysis of the projected DOS on orbitals indicates that the larger contributions are from the $\mathrm{O}$ and $\mathrm{Sb} p$ orbitals.

As consequence of the $\mathrm{O}$ doping, the optical properties are also affected. In Fig. 4 the difference between the absorption coefficients of the undoped and doped host is represented. For the two
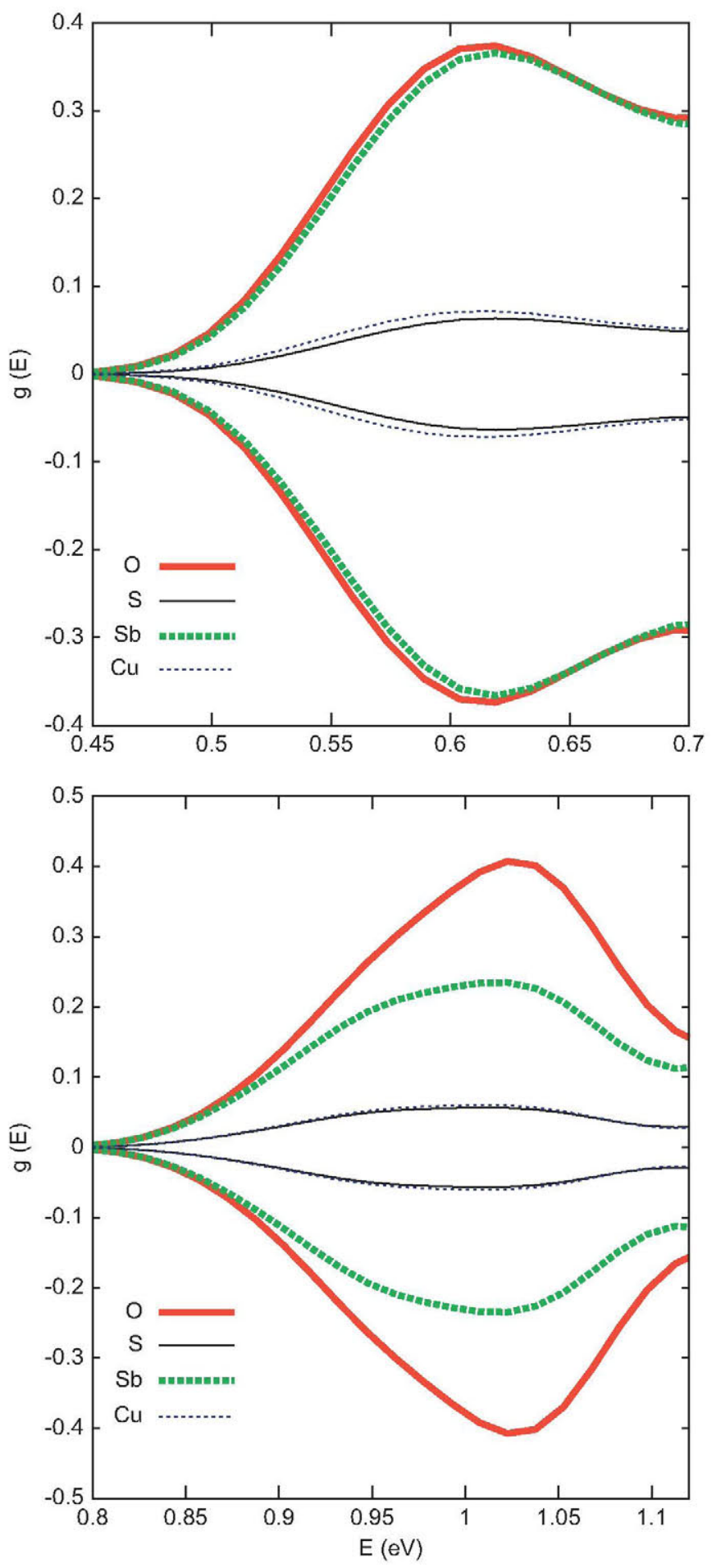

Fig. 3. Projected DOS on the atoms with GGA for O-doped $\mathrm{Cu}_{3} \mathrm{SbS}_{3}$ in the (a) Ws and (b) Ss. The VB edge has been chosen as the energy origin. 

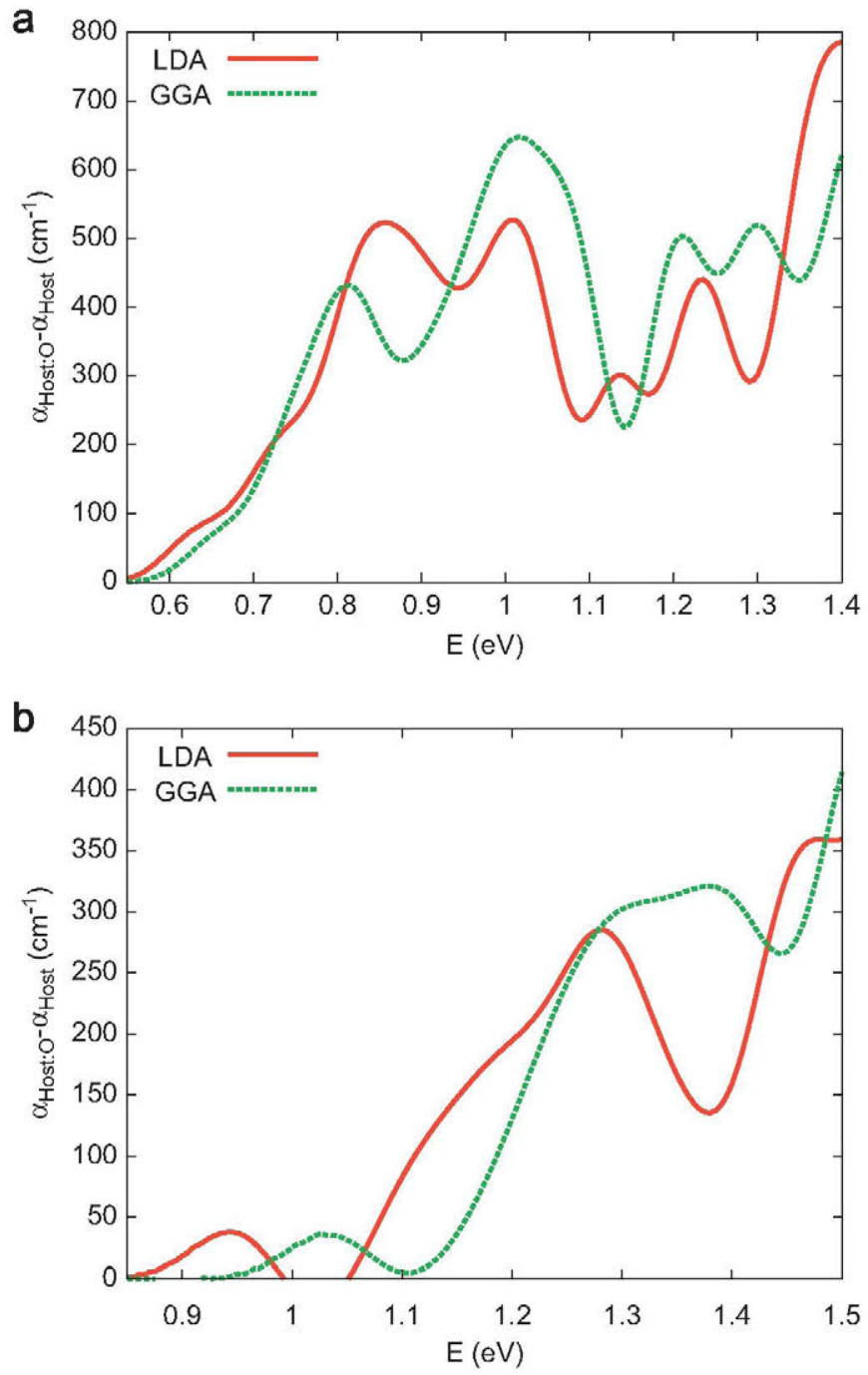

Fig. 4. Difference between the absorption coefficients of the O-doped host $\left(\alpha_{\text {Host:O}}\right)$ and undoped host $\left(\alpha_{\text {Host }}\right)$ for the (a) Ws, and (b) Ss.

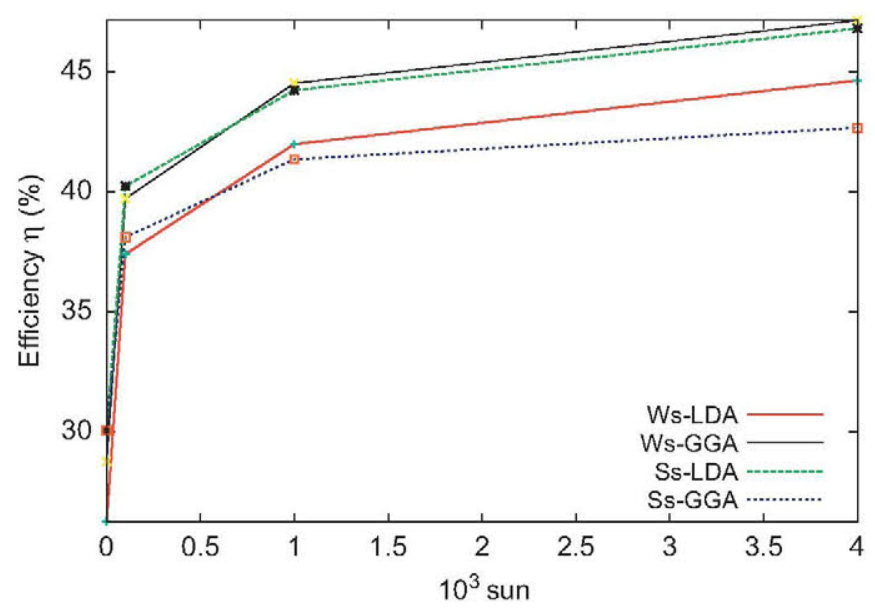

Fig. 5. Efficiency $\eta(\%)$ versus sunlight concentration $\left(10^{3}\right.$ sun) using the results of defect formation energy calculations with GGA and LDA.

structures, the $\mathrm{O}$ doping increases the absorption coefficient at the $\mathrm{CB}$ edge.

Using defect formation energy calculations, the energy position of the $\mathrm{O}$ acceptor level $\left(e_{A}\right)$ with respect to the $\mathrm{CB}$ edge
( $e_{C}-e_{A}$, where $e_{C}$ is the energy of the $\mathrm{CB}$ edge) for the Ws and $\mathrm{Ss}$ and with GGA and LDA are: $0.20 \mathrm{eV}$ (Ws-LDA), $0.25 \mathrm{eV}$ (Ws-GGA), $0.23 \mathrm{eV}$ (Ss-LDA), and $0.13 \mathrm{eV}$ (Ss-GGA). With these values, we have estimated the maximum efficiency for maximum solar concentration $\left(46,050 \mathrm{sun}, 1 \mathrm{sun}=1 \mathrm{~kW} / \mathrm{m}^{2}\right)$ according to the model described in Ref. [18]. The results are in Fig. 5. The maximum efficiency obtained for $\mathrm{Cu}_{3} \mathrm{SbS}_{3}: 0$ with GGA and LDA in the Ws and Ss are: $40 \%$ (Ws-LDA), $51.9 \%$ (Ws-GGA), $51.0 \%$ (SsLDA) and 46.3\% (Ss-GGA). These maxima are larger than the efficiency of a $\mathrm{Cu}_{3} \mathrm{SbS}_{3}$ single junction solar cell with equal solar concentration $(\sim 40 \%)$. For concentrations larger than approximately 100 sun, the efficiency of the 0 -doped $\mathrm{Cu}_{3} \mathrm{SbS}_{3}$ is larger than the maximum efficiency (for maximum solar concentration) of a $\mathrm{Cu}_{3} \mathrm{SbS}_{3}$ single junction solar cell.

\section{Conclusions}

In summary, we have used first-principles density functional methods in order to analyze the electronic properties of the host and O-doped $\mathrm{Cu}_{3} \mathrm{SbS}_{3}$ in both the Ws and Ss. We used both LDA and GGA exchange-correlation potentials. In order to obtain the gaps and the impurity levels, we used both single-particle energies and total energy defect calculations. An analysis of the single-particle band energies and of the total energy defect calculations indicates that substitution of $\mathrm{S}$ by $\mathrm{O}$ leads to an acceptor level very closer to the $\mathrm{CB}$. This acceptor level is mainly results from $\mathrm{Sb}$ and $\mathrm{O}$ orbital combinations.

Using the first-principles results we have estimated the maximum efficiency of this photovoltaic absorber material as a function of sunlight concentration. Above 100 sun, the efficiency of this compound is larger than that of host material with a single gap.

\section{Acknowledgments}

This work has been supported by the National Spanish projects CONSOLIDER (CSD2006-0004), Denquiband (PLE2009-0045), Bibiana (PIB2010US-00096) and Nanogefes (ENE2009-14481-C02-01), by the European Commission through the funding of the project IBPOWER (Ref. N: Grant Agreement 211640) and NGCPV (FP7-EUJPN 283798), and by La Comunidad de Madrid through the funding of the project NUMANCIA-2 (Ref. N: S-2009/ENE-1477).

\section{References}

[1] S. Manolache, A. Duta, L. Isac, M. Nanu, A. Goossens, J. Schoonman, The influence of the precursor concentration on $\mathrm{CuSbS}_{2}$ thin films deposited from aqueous solutions, Thin Solid Films 515 (2007) 5957-5960.

[2] A. Rabhi, M. Kanzari, B. Rezig, Optical and structural properties of $\mathrm{CuSbS}_{2}$ thin films grown by thermal evaporation method, Thin Solid Films 517 (2009) 2477-2480.

[3] Y. Rodríguez-Lazcano, M.T.S. Nair, P.K. Nair, $\mathrm{CuSbS}_{2}$ thin film formed through annealing chemically deposited $\mathrm{Sb}_{2} \mathrm{~S}_{3}$-CuS thin films, Journal of Crystal Growth 223 (2001) 399-406<http://www.sciencedirect.com/science/article/pii/S0022024801006728>.

[4] Y. Rodríguez-Lazcano, M.T.S. Nair, P.K. Nair, $\mathrm{Cu}_{x} \mathrm{SbyS}_{z}$ thin films produced by annealing chemically deposited Sb2S3-CuS thin films, Modern Physics Letters B 15 (2001) 667-670.

[5] S. Bloß, M. Jansen, Synthesis of microscale particles of ternary sulphides via an adjusted polyol-route, Zeitschrift fuer Naturforschung 58b (2003) 1075-1078.

[6] Changhua An, Ying Jin, Kaibin Tang, Yitai Qian, Selective synthesis and characterization of famatinite nanofibers and tetrahedrite nanoflakes, Journal of Materials Chemistry 13 (2003) 301-303.

[7] O. Madelung, Semiconductors: Data Handbook, Springer-Verlag 2000 (section 9.4).

[8] D.R. Lide (Ed.), Handbook of Chemistry and Physics, 90th edn., CRC Press, Taylor \& Francis Group, LLC, 2009-2010, pp. 12-88.

[9] A. Rabhi, M. Kanzari, B. Rezig, Growth and vacuum post-annealing effect on the properties of the new absorber $\mathrm{CuSbS}_{2}$ thin films, Materials Letters 62 (2008) 3576-3578. 
[10] J. Zhou, G.-Q. Bian, Q.-Y. Zhu, Y. Zhang, C.-Y. Li, J. Dai, Solvothermal crystal growth of $\mathrm{CuSbQ}_{2}(\mathrm{Q}=\mathrm{S}, \mathrm{Se})$ and the correlation between macroscopic morphology and microscopic structure, Journal of Solid State Chemistry 182 (2009) 259-264.

[11] D.W. Bullett, W.G. Dawson, Bonding relationships in some temary and quarternary phosphide and tetrahedrite structures: $\left(\mathrm{Ag}_{6} \mathrm{M}_{4} \mathrm{P}_{12}\right) \mathrm{M}_{6}$ ', $\mathrm{Cu}_{12+\chi} \mathrm{Sb}_{4} \mathrm{~S}_{13}$ and $\mathrm{Cu}_{14-\chi} \mathrm{Sb}_{4} \mathrm{~S}_{13}, \mathrm{Ln}_{6} \mathrm{Ni}_{6} \mathrm{P}_{17}$, Journal of Physics C: Solid State Physics 19 (1986) 5837-5847.

[12] C. Tablero, Acceptor and donor ionization energy levels in O-doped ZnTe, Computational Materials Science 49 (2010) 368-371.

[13] C. Tablero, A. Marti, A. Luque, Analyses of the intermediate energy levels in ZnTe:O alloys, Applied Physics Letters 96 (2010) 121104.

[14] B. Lee, L. Wang, Electronic structure of ZnTe:O and its usability for intermediate band solar cell, Applied Physics Letters 96 (2010) 071903.

[15] Y. Burki, P. Sshwendimann, W. Czaja, H. Berger, The temperature dependence of the photoluminescence and lifetime of ZnTe:O, Journal of Physics: Condensed Matter 5 (1993) 9235-9252;

Y. Burki, P. Sshwendimann, W. Czaja, H. Berger, Optical gain in ZnTe: 0 at $2 \mathrm{~K}$, Europhysics Letters 13 (1990) 555-559.

[16] W. Wang, A.S. Lin, J.D. Phillips, W.K. Metzger, Generation and recombination rates at ZnTe:O intermediate band states, Applied Physics Letters 95 (2009) 261107.

[17] J.L. Merz, Isoelectronic oxygen trap in ZnTe, Physical Review 176 (1968) 961-968;

W.K. Ge, S.B. Lam, I.K. Sou, J. Wang, Y. Wang, G.H. Li, H.X. Han, Z.P. Wang, Sulfur forming an isoelectronic center in zinc telluride thin films, Physical Review B 55 (1997) 10035-10039;

M.J. Seong, H. Alawadhi, l. Miotkowski, A.K. Ramdas, S. Miotkowska, Oxygen isoelectronic impurity in $\mathrm{ZnS}_{\alpha} \mathrm{Te}_{1-x}$, Physical ReviB 60 (1999) R16275-R16278;

Y.M. Yu, S. Nam, K.-S. Lee, Y. Dae Choi, O. Byungsung, Photoluminescence characteristics of ZnTe epilayers, Journal of Applied Physics 90 (2001) 807-812.

[18] A. Luque, A. Martí, Increasing the efficiency of ideal solar cells by photon induced transitions at intermediate levels, Physical Review Letters 78 (1997) 5014-5017.
[19] W. Kohn, L.J. Sham., Self-consistent equations including exchange and correlation effects, Physical Review 140 (1965) A1133-A1138.

[20] J.M. Soler, E. Artacho, J.D. Gale, A. García, J. Junquera, P. Ordejon, D. Sánchez-Portal, The SIESTA method for $a b$ initio order- $N$ materials simulation, Journal of Physics: Condensed Matter 14 (2002) 2745-2779, and references therein.

[21] J.P. Perdew, A. Zunger, Self-interaction correction to density-functional approximations for many-electron systems, Physical Review B 23 (1981) 5048-5079.

[22] D.M. Ceperley, B.J. Alder, Ground state of the electron gas by a stochastic method, Physical Review Letters 45 (1980) 566-569.

[23] N. Troullier, J.L. Martins, Efficient pseudopotentials for plane-wave calculations, Physical Review B 43 (1991) 1993-2006.

[24] L. Kleinman, D.M. Bylander, Efficacious form for model pseudopotentials, Physical Review Letters 48 (1982) 1425-1428; D.M. Bylander, L. Kleinman, 4 f resonances with norm-conserving pseudopotentials, Physical Review B 41 (1990) 907-912.

[25] O.F. Sankey, D.J. Niklewski, $A b$ initio multicenter tight-binding model for molecular-dynamics simulations and other applications in covalent systems, Physical Review B 40 (1989) 3979-3995.

[26] C.G. Van de Walle, J. Neugebauer, First-principles calculations for defects and impurities: applications to III-nitrides, Journal of Applied Physics 95 (2004) 3851 .

[27] J.E. Northrup, S.B. Zhang, Dopant and defect energetics: Si in GaAs, Physical Review B 47 (1993) 6791-6794.

[28] D.A. Drabold, S. Estreicher, Theory of Defects in Semiconductors, Springer, Berlin, Heidelberg, 2007, pp. 28-92.

[29] V. Kocman, E.W. Nuffield, The crystal structure of wittichenite, $\mathrm{Cu}_{3} \mathrm{BiS}_{3}$, Acta Crystallographica B B29 (1973) 2528-2535.

[30] A. Pfitzner, Disorder of $\mathrm{Cu}^{+}$in $\mathrm{Cu}_{3} \mathrm{SbS}_{3}$ : structural investigations of the highand low-temperature modification, Zeitschrift für Kristallographie 213 (1998) 228-236.

[31] H.J. Whitfield, Polymorphism in skinnerite, $\mathrm{Cu}_{3} \mathrm{SbS}_{3}$, Solid State Communication 33 (1980) 747-748.

[32] E. Makovicky, T. Balić-Žunić, The crystal structure of the skinnerite $\mathrm{P}_{2} / \mathrm{c}-\mathrm{Cu}_{3} \mathrm{SbS}_{3}$, from powder data, Canadian Mineralogist 33 (1995) 655-663. 